\title{
Growth of Inflaton Perturbations and the Post-Inflation Era in Supersymmetric Hybrid Inflation Models
}

\author{
MATt BRoAdheAd ${ }^{1}$ AND John MCDONALD ${ }^{2}$ \\ Dept. of Mathematical Sciences, University of Liverpool, Liverpool L69 3BX, England
}

October 31, 2018

\begin{abstract}
It has been shown that hybrid inflation may end with the formation of nontopological solitons of inflaton field. As a first step towards a fully realistic picture of the post-inflation era and reheating in supersymmetric hybrid inflation models, we study the classical scalar field equations of a supersymmetric hybrid inflation model using a semi-analytical ansatz for the spatial dependence of the fields. Using the minimal D-term inflation model as an example, the inflaton field is evolved using the full 1-loop effective potential from the slow-rolling era to the $U(1)_{F I}$ symmetry-breaking phase transition. Spatial perturbations of the inflaton corresponding to quantum fluctuations are introduced for the case where there is spatially coherent $U(1)_{F I}$ symmetry breaking. The maximal growth of the dominant perturbation is found to depend only on the ratio of superpotential coupling $\lambda$ to the gauge coupling $g$. The inflaton condensate fragments to nontopological solitons for $\lambda / g \gtrsim 0.09$. Possible consequences of non-topological soliton formation in fully realistic SUSY hybrid inflation models are discussed.
\end{abstract}

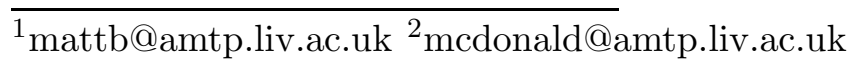




\section{Introduction}

An important event in the history of the Universe is the process by which it became hot after inflation, known as reheating. A full understanding of this epoch is essential for a full understanding of cosmology. It is also important to have a clear picture of the post-inflation cosmological enviroment against which ideas in particle physics and cosmology can be judged. The conventional view of reheating following inflation has been based on the perturbative decay of a spatially homogeneous Bose condensate of inflaton scalars [1]. This picture, however, has been challanged in recent years. It was firstly observed that the inflaton condensate might decay non-perturbatively via parametric resonance [2, 3, 4]. More recently, for some inflation models, in particular hybrid inflation [5], it was shown that the inflaton sector Bose condensate can be unstable with respect to spatial perturbations of the scalar fields [6, 7]. The growth of the spatial perturbations could either be slow, in which case a coherently oscillating condensate would form prior to spatial perturbations going non-linear ('inflaton condensate fragmentation') [6], or rapid, in which case the spatial perturbations dominate the total energy density before the inflaton can enter into coherent oscillations ('tachyonic preheating') [7. In the case of inflaton condensate fragmentation it was observed that the final state of the process is likely to be, at least for some range of parameters of the model, the formation of non-topological soliton-like objects ('inflaton condensate lumps') 6, 8]. The formation of such objects in hybrid inflation models was subsequently confirmed in lattice simulations [9. Recently it has been shown that inflaton condensate fragmentation can also occur in single field chaotic inflation models [10.

However, there has been to date no detailed study of condensate fragmentation and non-topological soliton formation in a fully realistic hybrid inflation model. Of particular interest is the case of supersymmetric (SUSY) hybrid inflation models, which are prime candidates for natural inflation models in the context of SUSY 11, 12. (The alternative, SUSY chaotic inflation, is disfavoured by the initial inflaton expectation value required, larger than the natural scale of supergravity (SUGRA) corrections, 
$M=M_{P l} / \sqrt{8 \pi}[13$.$) In the following we will focus on the case of the minimal D-$ term hybrid inflation model as a specific example. In D-term inflation models the energy density is provided by a Fayet-Illiopoulos D-term associated with a $U(1)_{F I}$ gauge symmetry [12. D-term inflation is particularly favoured as it provides a natural solution to the $\eta$-problem of SUGRA inflation models [14].

Hybrid inflation models in general are based on two scalar fields, an inflaton, $S$, and a second field, $\Phi$, which undergoes a symmetry-breaking phase transition which terminates inflation. (We will refer to these as the 'inflaton sector' fields.) As well as the homogeneous scalar fields considered in conventional D-term inflation models, there will also be quantum fluctuations leading to spatial perturbations of the scalar fields [1]. In order to establish the initial conditions for the growth of spatial perturbations following inflation, it is essential to follow the evolution of the inflaton from the slow-rolling epoch during inflation until the phase transition which terminates hybrid inflation. The evolution of the inflaton prior to the phase transition in SUSY hybrid inflation models is dominated by the 1-loop effective potential. Therefore in the following we will consider the evolution of the inflaton sector fields in D-term inflation including the full 1-loop radiative correction.

Spatial perturbations of the inflaton sector fields arise initially as a result of quantum fluctuations. A method to study the evolution of these fluctuations into the classical regime has been developed in [15]. So long as the final state of the evolution of the scalar fields is semi-classical, the evolution may be studied by solving the classical equations of motion but with a classical probability distribution for the initial conditions, determined by the initial quantum state of the field. (Following [15], we will refer to this as the 'equivalent classical stochastic field' (ECSF) method in the following.)

In this paper we study the evolution of spatial perturbations in D-term inflation using a semi-analytical model with simplified initial conditions for the spatial perturbations. This will allow us to gain a physical understanding of the process of spatial perturbation growth whilst providing an example against which future numerical simulations may be tested. We will argue that the growth of perturbations of the inflaton 
in the semi-analytical model represents the maximum possible growth in realistic models. In addition, we will show that that the formation of cosmic strings is likely to be rare on scales smaller than the horizon and so is unlikely to play a significant role in the subsequent evolution of the energy density, in which case the model we consider should give a good estimate of the growth of perturbations in the realistic case.

As in all previous studies, we will consider the evolution of a real inflaton field. However, in fully realistic models of SUSY inflation, the inflaton is a complex field. As such, none of the existing analyses of spatial perturbation growth in hybrid inflation models fully describes the SUSY case. One result of the complex nature of the inflaton field is that the non-topological solitons which form at the end of hybrid inflation are likely to be Q-balls [10, 23]. We will discuss how the analysis presented here relates to Q-ball formation and discuss some possible cosmological consequences of non-topological soliton formation in SUSY hybrid inflation models.

The paper is organised as follows. In Section 2 we discuss the D-term inflation model and the full 1-loop effective potential. In Section 3 we discuss the initial conditions used for the spatial perturbations. In Section 4 we discuss the semi-analytical ansatz used to model the growth of spatial perturbations. In Section 5 we discuss the evolution of perturbations of the inflaton sector fields in the semi-analytical model. In Section 6 we discuss some possible consequences of non-topological soliton formation in realistic SUSY hybrid inflation models. In Section 7 we present our conclusions. 


\section{Equations of Motion and 1-loop Effective Poten- tial}

In the following we will consider the minimal SUSY D-term hybrid inflation model ${ }^{1}$ [12. The superpotential of the minimal D-term inflation model is

$$
W=\lambda S \Phi_{+} \Phi_{-}
$$

The tree-level scalar potential is then

$$
V=\lambda^{2}|S|^{2}\left(\left|\Phi_{+}\right|^{2}+\left|\Phi_{-}\right|^{2}\right)+\lambda^{2}\left|\Phi_{+}\right|^{2}\left|\Phi_{-}\right|^{2}+\frac{g^{2}}{2}\left(\left|\Phi_{+}\right|^{2}-\left|\Phi_{-}\right|^{2}+\xi\right)^{2},
$$

where $S$ is the inflaton, $\Phi_{ \pm}$are fields with charges \pm 1 with respect to a Fayet-Illiopoulos $U(1)_{F I}$ gauge symmetry, $\xi>0$ is the Fayet-Illiopoulos gauge term $\left(\xi^{1 / 2} \approx 8.5 \times\right.$ $10^{15} \mathrm{GeV}[16]$ ) and $g$ is the $U(1)_{F I}$ gauge coupling. Since the minimum of the potential as a function of $S$ is generally at $\left|\Phi_{+}\right|=0$ and since $\left|\Phi_{+}\right|=0$ at the end of inflation, we will assume this value throughout. (We will discuss this assumption later.) We will also consider the scalar potential along the real $S$ and $\Phi_{-}$directions. Thus with $s=\sqrt{2} \operatorname{Re}(S)$ and $\phi_{-}=\sqrt{2} \operatorname{Re}\left(\Phi_{-}\right)$, the tree-level scalar potential becomes

$$
V\left(s, \phi_{-}\right)=\frac{\lambda^{2}}{4} s^{2} \phi_{-}^{2}+\frac{g^{2}}{2}\left(\xi-\frac{\phi_{-}^{2}}{2}\right)^{2} .
$$

The $U(1)_{F I}$ symmetry breaking transition occurs once $s<s_{c} \equiv \sqrt{2} g \xi^{1 / 2} / \lambda$.

In addition, we require the 1-loop effective potential as a function of $s$ and $\phi_{-}$, $\Delta V\left(s, \phi_{-}\right)$. This has been done previously for the case where $\phi_{-}$is fixed at the minimum of its potential as a function of $s[17$. However, as we wish to discuss the equations of motion of the $s$ and $\phi_{-}$field seperately here, we require the mass eigenvalues and effective potential as a function of both fields. For $s<s_{c}$ the scalar and gauge boson mass terms and degrees of freedom as a function of $s$ and $\phi_{-}$are given in Table 1.

\footnotetext{
${ }^{1}$ The tree-level scalar potential for the D-term inflation model becomes equivalent to the minimal F-term inflation model [1] in the limit where $\lambda=\sqrt{2} g$. Therefore study of the D-term model should also give an insight into the growth of spatial perturbations in the F-term inflation model.
} 


\begin{tabular}{|c|c|c|}
\hline Field & d.o.f & Mass Squared \\
\hline$\eta_{+}$ & 1 & $\frac{1}{2}\left[B+\sqrt{B^{2}-4 C}\right]$ \\
$\eta_{-}$ & 1 & $\frac{1}{2}\left[B-\sqrt{B^{2}-4 C}\right]$ \\
$s_{2}$ & 1 & $\frac{\lambda^{2}}{2} \phi_{-}^{2}$ \\
$\Phi_{+}$ & 2 & $\frac{\lambda^{2}}{2} s^{2}+\left(\frac{\lambda^{2}-g^{2}}{2}\right) \phi_{-}^{2}+g^{2} \xi$ \\
$A$ & 3 & $g^{2} \phi_{-}^{2}$ \\
\hline
\end{tabular}

Table 1: Mass terms and physical degrees of freedom for $s<s_{c}$.

Here $B=\frac{1}{2} \lambda^{2}\left(s^{2}+\phi_{-}^{2}\right)+\frac{3}{2} g^{2} \phi_{-}^{2}-g^{2} \xi$ and $C=\frac{1}{2} \lambda^{2} \phi_{-}^{2}\left(\frac{1}{2} \lambda^{2} s^{2}+\frac{3}{2} g^{2} \phi_{-}^{2}-g^{2} \xi\right)-$ $\frac{1}{4} \lambda^{4} s^{2} \phi_{-}^{2} . \eta_{ \pm}$are the eigenstates of the $s_{1}, \phi_{1}$ mass matrix. (We define $S=\left(s_{1}+i s_{2}\right) / \sqrt{2}$ and $\Phi_{-}=\left(\phi_{1}+i \phi_{2}\right) / \sqrt{2}$.) $A$ is the $U(1)_{F I}$ gauge boson, with $\phi_{2}$ the corresponding Goldstone boson ${ }^{2}$.

The fermion mass eigenstates come from the mass matrix $M_{\Psi}$ for $\Psi \equiv\left(\chi, \lambda_{+}, \lambda_{-}, \lambda_{S}\right)$, where $\chi$ is the $U(1)_{F I}$ gaugino, $\lambda_{+}\left(\lambda_{-}\right)$is the fermionic component of $\Phi_{+}\left(\Phi_{-}\right)$and $\lambda_{S}$ is the fermionic component of the $S$ chiral superfield,

$$
\Psi^{T} M_{\Psi} \Psi \equiv\left(\begin{array}{llll}
\chi & \lambda_{-} & \lambda_{+} & \lambda_{S}
\end{array}\right)\left[\begin{array}{cccc}
0 & g \phi_{-} & 0 & 0 \\
g \phi_{-} & 0 & \frac{\lambda}{\sqrt{2}} s & 0 \\
0 & \frac{\lambda}{\sqrt{2}} s & 0 & \frac{\lambda}{\sqrt{2}} \phi_{-} \\
0 & 0 & \frac{\lambda}{\sqrt{2}} \phi_{-} & 0
\end{array}\right]\left(\begin{array}{c}
\chi \\
\lambda_{-} \\
\lambda_{+} \\
\lambda_{S}
\end{array}\right) .
$$

The resulting eigenstates correspond to two Dirac fermons, of mass

$$
m_{1,2}=\frac{1}{2}\left[\frac{\lambda^{2}}{2} s^{2}+\left(g^{2}+\frac{\lambda^{2}}{2}\right) \phi_{-}^{2}\right] \pm \sqrt{\Delta},
$$

where

$$
\Delta=\frac{1}{4}\left(\left[\left(g^{2}+\frac{\lambda^{2}}{2}\right) \phi_{-}^{2}+\frac{\lambda^{2}}{2} s^{2}\right]^{2}-2 \lambda^{2} g^{2} \phi^{4}\right) .
$$

For $s>s_{c}$ there are two massive complex scalars $\Phi_{ \pm}$and a single Dirac fermion $\Psi$, with squared masses given in Table 2 .

\footnotetext{
${ }^{2}$ More precisely, $\Phi_{-}=\frac{\phi}{\sqrt{2}} e^{i \theta}$, with $\theta$ the Goldstone boson degree of freedom. The mass terms of the physical scalar field $\phi$ with $\theta=0$ are the same as those of $\phi_{1}$ with $\phi_{2}=0$.
} 


\begin{tabular}{|c|c|c|}
\hline Field & d.o.f & Mass Squared \\
\hline$\Phi_{+}$ & 2 & $\frac{\lambda^{2}}{2} s^{2}+g^{2} \xi$ \\
$\Phi_{-}$ & 2 & $\frac{\lambda^{2}}{2} s^{2}-g^{2} \xi$ \\
$\Psi$ & 4 & $\frac{\lambda^{2}}{2} s^{2}$ \\
\hline
\end{tabular}

Table 2: Mass terms and physical degrees of freedom for $s>s_{c}$.

The 1-loop effective potential as a function of $s$ and $\phi$ is given by the ColemanWeinberg formula

$$
\Delta V(s, \phi)=\frac{1}{64 \pi^{2}} \sum_{i}(-1)^{F} m_{i}^{4} \ln \left(\frac{m_{i}^{2}}{\Lambda^{2}}\right),
$$

where $\Lambda$ is a renormalisation scale and the contribution is negative for fermions. An important question is how this should be interpreted when a scalar mass squared becomes negative for small field expectation values. This problem also arises in the case of the Standard Model Higgs effective potential. Weinberg and $\mathrm{Wu}$ 18 argue that only the real part of the effective potential corresponds to a correction to the energy density and so enters the equations of motion. Thus if $m_{i}^{2}<0$, we can simply substitute $m_{i}^{2}=\left|m_{i}^{2}\right| e^{i \pi}$ in Eq. (77), in which case the real part of $\Delta V$ corresponds to replacing $m_{i}^{2}$ by $\left|m_{i}^{2}\right|$ throughout. We will use this proceedure in the following.

The full equations of motion to 1-loop are then

$$
\ddot{s}+3 H \dot{s}-\frac{\nabla^{2}}{a^{2}} s=-\frac{\lambda^{2} \phi_{-}^{2} s}{2}-\frac{\partial \Delta V}{\partial s},
$$

and

$$
\ddot{\phi}_{-}+3 H \dot{\phi}_{-}-\frac{\nabla^{2}}{a^{2}} \phi_{-}=-\frac{\lambda^{2} s^{2} \phi_{-}}{2}+g^{2}\left(\xi-\frac{\phi_{-}^{2}}{2}\right) \phi_{-}-\frac{\partial \Delta V}{\partial \phi_{-}} .
$$

\section{Spatial Perturbations and Initial Conditions}

\subsection{Equivalent Classical Stochastic Field}

We are interested in the growth of spatial perturbations of the scalar fields. These have their origin in quantum fluctuations. In studying the growth of quantum fluctuations of 
a scalar field, the method used is that of the equivalent classical stochastic field (ECSF) [15. This is based on the observation that for quantum states which evolve to semiclassical final states, expectation values in the full quantum field theory are the same as those obtained by solving the classical equations of motion with initial conditions which have a classical stochastic distribution determined by the initial quantum state ${ }^{3}$. For the case we are considering here, the initial state is taken to correspond to the conformal vacuum state of a massless scalar field $\phi$ in (quasi) de Sitter space $(H \approx$ constant). The corresponding initial conditions for the field modes are [15]

$$
\begin{gathered}
|y(\mathbf{k})|^{2}=\frac{1}{2 k}\left(1+\frac{1}{k^{2} \eta^{2}}\right) \\
|p(\mathbf{k})|^{2}=\left(\frac{1}{4 k^{2} \eta^{2}}\right) \frac{1}{|y(\mathbf{k})|^{2}}=\frac{1}{2 k \eta^{2}}\left(1+\frac{1}{k^{2} \eta^{2}}\right)^{-1} .
\end{gathered}
$$

Here we have used the notation of [15], where $y(\mathbf{k})$ is the Fourier transform of the perturbation of the scalar field $\phi(\mathbf{x}), \delta \phi(\mathbf{x}) \equiv y(\mathbf{x}) / a$ (time dependence not explicitly stated), and we are considering initial conditions taken at scale factor $a=1 . p(\mathbf{k})$ is the Fourier transform of $p(\mathbf{x})$, the conjugate variable of $y(\mathbf{x})$

$$
p(\mathbf{x})=y^{\prime}-\left(\frac{a^{\prime}}{a}\right) y
$$

where a prime denotes differentiation with respect to conformal time $\eta\left(\eta=\int d t / a=\right.$ $\left.-(a H)^{-1}\right)$. These modes refer to Fourier transforms defined by

$$
\begin{aligned}
& y(\mathbf{x})=\frac{V^{1 / 2}}{(2 \pi)^{3 / 2}} \int y(\mathbf{k}) e^{-i \mathbf{k} \cdot \mathbf{x}} d^{3} k, \\
& y(\mathbf{k})=\frac{V^{-1 / 2}}{(2 \pi)^{3 / 2}} \int_{V} y(\mathbf{x}) e^{i \mathbf{k} \cdot \mathbf{x}} d^{3} x,
\end{aligned}
$$

where we retain an explicit spatial volume $V$ in order to account for the mass dimensions. For a real $y(\mathbf{x})$ we have $y(\mathbf{k})=y^{*}(-\mathbf{k})$.

Given the initial conditions, the subsequent evolution of the system is obtained by evolving the scalar field classically. In practice the important quantities are the

\footnotetext{
${ }^{3} \mathrm{~A}$ simple and useful illustration of this concept in the context of quantum mechanics is given in
} [19. 
root mean square (r.m.s.) spatial averages of the field fluctuations. For example, once the r.m.s. fluctuation of the field amplitude is of the order of the homogeneous field amplitude, the field will almost entirely exist in the overdense regions and therefore we expect the spatial perturbations to go non-linear and to fragment to non-topological solitons. The mean squared fluctuation is given by

$$
\left\langle y(\mathbf{x})^{2}\right\rangle=4 \pi \int k^{3}|y(\mathbf{k})|^{2} \frac{d k}{k}
$$

where $k=|\mathbf{k}|$. Thus modes with $|\mathbf{k}|$ in a range $\Delta k \sim k$ around $k$ will contribute to the r.m.s. fluctuation squared an amount [1]

$$
\Delta_{k}^{2} \equiv\left\langle y(\mathbf{x})^{2}\right\rangle_{k} \approx 4 \pi k^{3}|y(\mathbf{k})|^{2}
$$

We will model the classical evolution of the r.m.s. fluctuation due to modes with $|\mathbf{k}| \sim k$ by considering a single mode with $|\mathbf{k}|=k$ and initial amplitude given by $\Delta_{k}$. We will be particularly interested in the dominant mode (the largest at late times), since its wavelength is likely to determine the size of the non-topological solitons which form once the inflaton sector energy density perturbations become non-linear [6. 8. (This is similar to what is observed numerically in the case of a complex scalar condensate and Q-ball formation [20, 21.) The modes of interest in the following will have wavelength small compared with the horizon at the end of inflation. In this case $|k \eta|=k / H \gg 1$ and $|y(\mathbf{k})| \approx 1 / \sqrt{2 k}$. Then $\Delta_{k} \approx \sqrt{2 \pi} k$.

The momentum conjugate to $y(\mathbf{x})$ in the limit $k \gg H$ is given by

$$
p(\mathbf{x})=a(\dot{y}(\mathbf{x})-H y(\mathbf{x}))
$$

where 'dot' denotes differentiation with respect to time. Thus if $\dot{y}(\mathbf{x}) / y(\mathbf{x}) \equiv \delta \dot{\phi}(\mathbf{x}) / \delta \phi(\mathbf{x}) \gtrsim H$ (as expected if perturbation growth is significant) we have initially (at $a=1) p(\mathbf{x}) \approx$ $\dot{y}(\mathbf{x}) \equiv \delta \dot{\phi}(\mathbf{x})$. Therefore the initial mean squared rate of change of $\delta \phi(\mathbf{x})$ corresponding to the range $\Delta k \sim k$ around $k$ is related to $p(\mathbf{k})$ by (cf. Eq. (16)),

$$
\left\langle\delta \dot{\phi}^{2}(\mathbf{x})\right\rangle_{k} \approx 4 \pi k^{3}|p(\mathbf{k})|^{2}=2 \pi k^{4}\left(\frac{H}{k}\right)^{2}
$$


Thus to model the ECSF initial conditions we will consider the evolution of a scalar field mode $\delta \phi_{k}(\mathbf{x})$ with initially (at $t=t_{o}$ )

$$
\delta \phi_{k}\left(\mathbf{x}, t_{o}\right)=A\left(t_{o}\right) \sin (\mathbf{k} \cdot \mathbf{x})
$$

where

$$
A\left(t_{o}\right)=\sqrt{2 \pi} k
$$

and

$$
\left|\dot{A}\left(t_{o}\right)\right|=\sqrt{2 \pi} k^{2}\left(\frac{H}{k}\right)
$$

(In practice we find that $\left|\dot{A}\left(t_{o}\right)\right|$ has a negligible effect.)

We next consider the time at which the ECSF initial conditions should be applied. They strictly apply for the quantum vacuum state of an effectively massless scalar field $(m \ll H)$ in de Sitter space. For the inflaton, $s$, we may apply the initial condition at any time during slow rolling. So long as there is little growth of the effectively classical $s$ fluctuation from this time until $s$ reaches $s_{c}$ (which may be expected as there is only the 1-loop effective potential at this time) the same initial perturbation may be applied at $s_{c}$ (or more generally up to the time at which the $U(1)_{F I}$ symmetry breaking field develops a classical expectation value and the $s$ perturbation begins to grow significantly). At $s_{c}$ the $\phi_{-}$field is also massless. (However, there is a $\phi_{-}^{4}$ potential term which will limit the amplitude of effectively massless fluctuations to $V^{\prime \prime}\left(\phi_{-}\right) \lesssim H^{2}$.) Thus a natural time to apply the equivalent classical initial conditions to both $s$ and $\phi_{-}$would be at $s(t) \approx s_{c}$.

At $s_{c}$ the mean value of the $\phi_{-}$field is zero. The $U(1)_{F I}$ symmetry breaking occurs as a result of the growth of the $\phi_{-}$quantum equivalent classical fluctuations when the $\phi_{-}$field acquires a negative mass squared term once $s<s_{c}$. The amplitude of the quantum equivalent classical fluctuation is $\delta \phi_{-q} \approx \sqrt{2 \pi} k$. We first note that the largest value of $k$ for $\phi_{-}$fluctuations for which the $\phi_{-}$field can be considered as effectively massless corresponds to $V^{\prime \prime}\left(\delta \phi_{-q}\right)\left(\equiv 3 g^{2} \delta \phi_{-q}^{2} / 2\right) \approx H^{2}$. Thus the spectrum of equivalent classical fluctuations has an upper limit, $k^{2} \underset{\sim}{\sim} H^{2} / 3 \pi g^{2}$, beyond which the quantum fluctuations will be suppressed by the effective mass term. A 
fluctuation can grow due to the negative mass squared term so long as its physical momentum satisfies $k^{2}<\left|m_{\phi_{-}}^{2}\right|$, where $m_{\phi_{-}}^{2}=\left(\frac{\lambda^{2} s^{2}}{2}-g^{2} \xi\right)$. In addition, the mode can grow only once $\left|m_{\phi_{-}}^{2}\right| \gtrsim H^{2}$. Therefore, the largest amplitude mode which first begins to grow classically corresponds to $k^{2} \approx H^{2}$, which, for natural values of the $U(1)_{F I}$ gauge coupling not much smaller than 1 , is of the same order as the upper limit of the spectrum of equivalent classical fluctuations. Thus we may consider the dominant mode to correspond to $k \approx H$ and to start growing once $\left|m_{\phi_{-}}^{2}(s)\right| \approx H^{2}$, defined to occur at $s=s_{1}$. Once the $\phi_{-}^{4}$ interaction term is included, the minimum of the potential in the $\phi_{-}$direction for a given $s$ is at $\phi_{\min }=\sqrt{2}\left|m_{\phi_{-}}\right| / g$. Therefore the minimum of the potential is at around the amplitude of the largest quantum equivalent classical fluctuation which is able to grow due to the negative mass squared term. Once a mode is given a classical value of the order of $\phi_{\min }$, numerical solution of the classical equations of motion shows that a growing mode will remain close to $\phi_{\text {min }}(s)$ as $s$ decreases (Fig 1). This indicates that at any given time once $s<s_{1}$, the dominant quantum equivalent classical $\phi_{-}$mode will have amplitude around $\phi_{\min }(s)$.

This also indicates that the size of the domains of $U(1)_{F I}$ symmetry breaking and so seperation of the cosmic strings, which corresponds to the dominant $\phi_{-}$fluctuation with $k \approx H$, will not be much smaller than the horizon $\left(\lambda \approx 2 \pi / k \approx H^{-1}\right)$. In particular, the spacing of the cosmic strings will be much larger than the dynamical length scale of the inflaton sector fields, of the order of $\xi^{-1 / 2}$. Therefore we expect that cosmic strings will be relatively rare and that they will not play a significant role in the subsequent evolution of the inflaton sector fields and energy density.

\subsection{Initial Conditions}

In order to analyse the growth of $s$ perturbations in the presence of cosmic strings, it is necessary to perform a full lattice simulation. Here we wish to consider a simplified model without cosmic strings, which we have argued will not play a significant role in the growth of inflaton sector perturbations. This is achieved by correlating the direction of the initial $\phi_{-}$quantum fluctuations. Thus we will consider an initial perturbation of the $\phi_{-}$field with the same magnitude as the quantum equivalent 


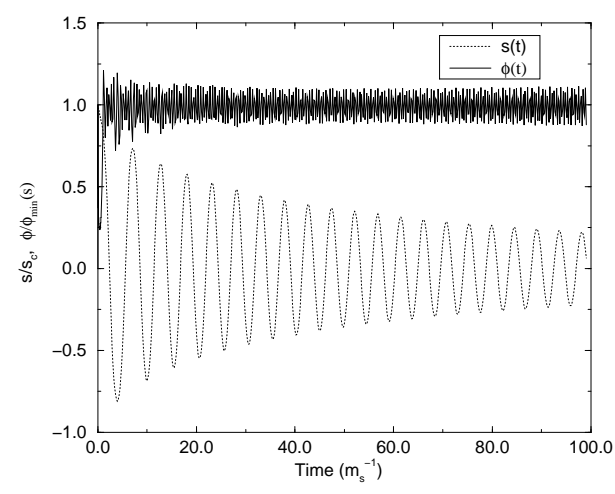

(a) $g=1.0$

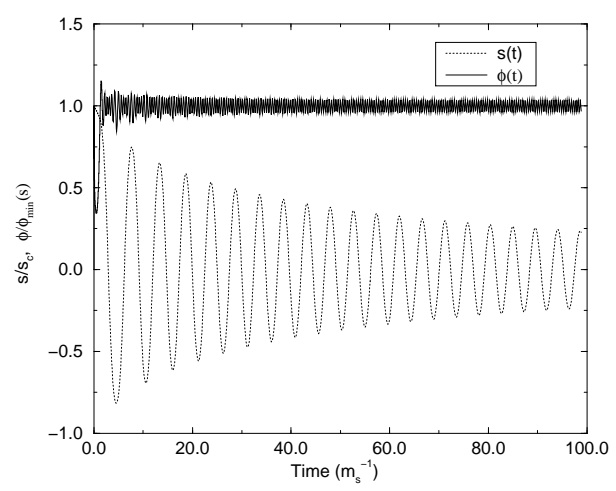

(b) $g=0.5$

Figure 1: After its initial perturbation $\phi$ tracks its minimum $\phi_{\min }(s)$ as a function of $s$ as $s$ decreases for both values of $g$. (Using $\frac{\lambda}{g}=0.14$ and $k=0.001 s_{c}$.)

classical fluctuations (i.e. of the order of $\phi_{\min }(s)$ at $s_{1}$ ) but homogeneous in space. We will refer to these as 'no cosmic string' (NCS) initial conditions in the following. The NCS initial conditions we consider are then

$$
\begin{gathered}
s=s_{1}, \\
\delta s=\sqrt{2 \pi} k \quad ; \quad|\delta \dot{s}|=\sqrt{2 \pi} k^{2}\left(\frac{H}{k}\right), \\
\phi_{-}=\phi_{\min }\left(s_{1}\right)
\end{gathered}
$$

and

$$
\delta \phi_{-}=0 ;\left|\delta \dot{\phi}_{-}\right|=0
$$

with $s_{1}$ corresponding to $\left|m_{\phi}\left(s_{1}\right)\right|^{2} \approx H^{2}$. We have set $\delta \phi_{-}=0$ initially since the quantum equivalent classical $\phi_{-}$perturbation has been rotated in space to become homogeneous. The initial value of the rate of rolling of the homogeneous inflaton field, $\dot{s}$, which plays an important role in determining the subsequent growth of the spatial perturbations, is obtained by evolving the homogeneous $s$ field from the slow-rolling regime at $s \gg s_{c}$ to $s_{1}$ using the 1-loop effective potential. 
The NCS initial conditions we have introduced are not strictly physical. Nevertheless, the resulting growth of the inflaton perturbation has a general physical interpretation, namely it is the maximum possible growth. This follows since the classical growth of inflaton perturbations is due to the non-zero $\phi_{-}$expectation value as a function of $s$; in the limit $\phi_{-}=0$ there is no significant growth since $s$ then only experiences a 1-loop effective potential as in the case $s>s_{c}$. The effect of the cosmic strings is to reduce the value of $\phi_{-}$at a given point in space relative to the case with no cosmic strings (the $U(1)_{F I}$ symmetry breaking field vanishing at the centre of the strings), so reducing the degree of $U(1)_{F I}$ symmetry breaking on average. Therefore we expect the growth of inflaton perturbations to be suppressed relative to the case with no cosmic strings. For the particular case of D-term inflation we have also argued that cosmic strings should be relatively rare on sub-horizon scale and so should not play a major role in determining the evolution of the energy density in the bulk between the strings. In this case the NCS initial conditions should be a good approximation to the fully realisitic case.

\section{Semi-Analytical Model for Spatial Perturbation Growth}

In this section we consider the growth of spatial perturbations using an analytical ansatz for the spatial dependence of the perturbation modes. Our method will be to study the evolution of a single perturbation mode of the $s$ and $\phi_{-}$field, using an ansatz for the functional form of the space dependence of the fields. We first discuss the ansatz for a generic real scalar field $\Phi$. The field we consider is assumed to initially have the form $\Phi(\mathbf{x}, t)=\Phi(t)+\delta \Phi(t) \sin (\mathbf{k} \cdot \mathbf{x})$, where $\Phi(t)$ is the homogeneous field. In general, we would expect the spatial position of the extrema of $\Phi(\mathbf{x}, t)$ at later times to remain the same as their initial positions. Thus we expect that $\Phi(\mathbf{x}, t)=$ $\Phi(t)+f(t) g(\mathbf{x})$, where $g(\mathbf{x})$ has the same periodicity in space as the initial perturbation. If the growth of the perturbation was strictly linear, then the correct choice would be $g(\mathbf{x})=\sin (\mathbf{k} \cdot \mathbf{x})$. However, it is possible that non-linearity could play a role in 
the growth of the perturbation, in which case this assumption must be considered an ansatz, whose validity depends on how well the true interpolating function $g(\mathbf{x})$ between the maxima and minima of $\Phi(\mathbf{x}, t)$ in space can be modelled by $\sin (\mathbf{k} . \mathbf{x})$. The match need not be exact for the physics of perturbation growth to be reasonably well modelled.

The equation of motion of $\Phi$ is

$$
\ddot{\Phi}+3 H \dot{\Phi}-\frac{\nabla^{2}}{a^{2}} \Phi=-\frac{\partial V(\Phi)}{\partial \Phi}
$$

We introduce the ansatz $\Phi(\mathbf{x}, t)=\Phi(t)+\delta \Phi(t) \sin (\mathbf{k} \cdot \mathbf{x})$, in which case the equations of motion become

$$
\ddot{\Phi}+3 H \dot{\Phi}+\frac{\mathbf{k}^{2}}{a^{2}} \delta \Phi \sin (\mathbf{k} \cdot \mathbf{x})=-\frac{\partial V(\Phi)}{\partial \Phi}
$$

Let $\Phi_{+}$and $\Phi_{-}$be values of $\Phi(\mathbf{x}, t)$ at points in space $(\sin (\mathbf{k} \cdot \mathbf{x})= \pm 1)$ corresponding initially to the maximum and minimum of $\Phi(\mathbf{x}, t)$. Thus $\Phi_{+}=\Phi(t)+\delta \Phi(t)$ and $\Phi_{-}=\Phi(t)-\delta \Phi(t)$. The equations at these points in space are then

$$
\ddot{\Phi}_{+}+3 H \dot{\Phi}_{+}+\frac{\mathbf{k}^{2}}{a^{2}} \delta \Phi=-\left.\frac{\partial V(\Phi)}{\partial \Phi}\right|_{\Phi_{+}}
$$

and

$$
\ddot{\Phi}_{-}+3 H \dot{\Phi}_{-}-\frac{\mathbf{k}^{2}}{a^{2}} \delta \Phi=-\left.\frac{\partial V(\Phi)}{\partial \Phi}\right|_{\Phi_{-}} .
$$

Thus with $\delta \Phi=\left(\Phi_{+}-\Phi_{-}\right) / 2$ the equations for the evolution of the field at points in space corresponding to maxima and minima of $\Phi$ are

$$
\ddot{\Phi}_{+}+3 H \dot{\Phi}_{+}+\frac{\mathbf{k}^{2}}{2 a^{2}}\left(\Phi_{+}-\Phi_{-}\right)=-\left.\frac{\partial V(\Phi)}{\partial \Phi}\right|_{\Phi_{+}}
$$

and

$$
\ddot{\Phi}_{-}+3 H \dot{\Phi}_{-}-\frac{\mathbf{k}^{2}}{2 a^{2}}\left(\Phi_{+}-\Phi_{-}\right)=-\left.\frac{\partial V(\Phi)}{\partial \Phi}\right|_{\Phi_{-}} .
$$

Thus we have only to solve two coupled equations which are purely functions of $t$. We will refer to this as the semi-analytical model for perturbation growth.

We can generalise the semi-analytical model to the case of hybrid inflation with two scalar fields. We consider an ansatz of the form $s(\mathbf{x}, t)=s(t)+\delta s(t) \sin (\mathbf{k} \cdot \mathbf{x})$ 
and $\phi(\mathbf{x}, t)=\phi(t)+\delta \phi(t) \sin (\mathbf{k} \cdot \mathbf{x})$. At $s(t)=s_{1}$ the $\phi$ field at a point in space corresponding to the minimum of $s(\mathbf{x}, t), s_{-}$, will experience the largest symmetry breaking negative mass squared term. Therefore at this point $\phi(\mathbf{x}, t)$ begins to grow first, corresponding to $\phi_{+}$. (In accordance with NCS boundary conditions, we assume the symmetry breaking direction is correlated.) We therefore denote the fields at this point in space by $\left(s_{-}, \phi_{+}\right)$, whilst the fields at the maximum of $s(\mathbf{x}, t)$ are $\left(s_{+}, \phi_{-}\right)$. (The spatial dependence of the $s$ perturbation is therefore imprinted on the $\phi$ field.) This is illustrated in Figure 2. The semi-analytic equations are then

$$
\begin{gathered}
\ddot{s}_{+}+3 H \dot{s}_{+}+\frac{k^{2}}{2 a^{2}}\left(s_{+}-s_{-}\right)=-\left.\frac{\partial V(s, \phi)}{\partial s}\right|_{\left(s_{+}, \phi_{-}\right)}, \\
\ddot{\phi}_{-}+3 H \dot{\phi}_{-}-\frac{k^{2}}{2 a^{2}}\left(\phi_{+}-\phi_{-}\right)=-\left.\frac{\partial V(s, \phi)}{\partial \phi}\right|_{\left(s_{+}, \phi_{-}\right)},
\end{gathered}
$$

and

$$
\begin{aligned}
& \ddot{s}_{-}+3 H \dot{s}_{-}-\frac{k^{2}}{2 a^{2}}\left(s_{+}-s_{-}\right)=-\left.\frac{\partial V(s, \phi)}{\partial s}\right|_{\left(s_{-}, \phi_{+}\right)}, \\
& \ddot{\phi}_{+}+3 H \dot{\phi}_{+}+\frac{k^{2}}{2 a^{2}}\left(\phi_{+}-\phi_{-}\right)=-\left.\frac{\partial V(s, \phi)}{\partial \phi}\right|_{\left(s_{-}, \phi_{+}\right)} .
\end{aligned}
$$

Then $\delta s=\left(s_{+}-s_{-}\right) / 2$ and $\delta \phi=\left(\phi_{+}-\phi_{-}\right) / 2$, whilst the homogeneous mean fields are given by $s(t)=\left(s_{+}+s_{-}\right) / 2$ and $\phi(t)=\left(\phi_{+}+\phi_{-}\right) / 2$.

\section{Results for Perturbation Growth}

We next investigate the growth in inflaton perturbations using the NCS initial conditions and the semi-analytical ansatz for the evolution of the spatial perturbations. We will focus on the case $g=1$ but include $g=0.5$ for comparison.

In the figures we show the evolution of the homogeneous mean field $s(t)$ and the amplitude of spatial perturbation $\delta s(t)=\left(s_{+}-s_{-}\right) / 2$ for the semi-analytical model

using the D-term hybrid inflation scalar potential with 1-loop corrections. (For clarity we show the magnitude of $\delta s(t)$ in the figures, such that $\delta s(t)$ is always positive.) 


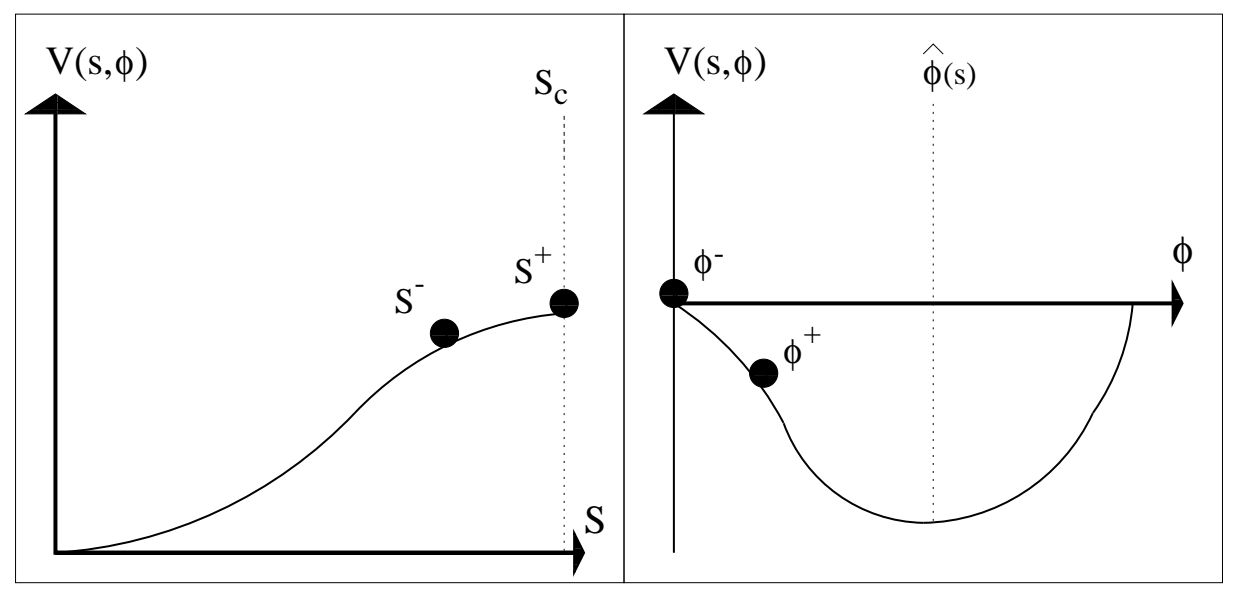

Figure 2: Schematic illustration of the time evolution of $\phi$ and $s$ as the inflaton $s$ passes from slow-roll phase to $U(1)_{F I}$ symmetry breaking phase.

The homogeneous inflaton field is evolved from the slow-rolling regime with initially $s \approx 20 s_{c}$ whilst the NCS boundary conditions for the perturbations are introduced at $s_{1}$. We expect that the growth of the spatial perturbations will become fully nonlinear with the formation of non-topological solitons ('fragmentation') once the spatial perturbation amplitude is similar to the homogeneous mean field, $\delta s(t) / s(t) \approx 1$.

In Figure 3 and 4 we show the evolution of $\delta s / s(t)$ for a mode of fixed wavenumber $k=0.01 s_{c}$ as $\lambda / g$ is increased for the case $g=1$. (We consider $a=1$ at $s=s_{c}$, such that $k$ is the physical wavenumber at this time.) In Figure 3a) with $\lambda / g=0.1$ there is little growth of $\delta s(t)$ whilst the mean field amplitude $s(t)$ decreases due to expansion. In Figure $3 \mathrm{~b}$ ) with $\lambda / g=0.12$ significant growth of the spatial perturbation begins to be seen. In Figure 4a) with $\lambda / g=0.14$ we see that the spatial perturbation amplitude increases rapidly to become equal to the mean field, $\delta s(t) / s(t) \approx 1$, in approximately one oscillation cycle of the homogeneous field. Finally, in Figure $4 \mathrm{~b}$ ) with $\lambda / g=0.2$ the spatial perturbation grows more rapidly and dominates the homogeneous field at all times after about one oscillation cycle of the homogeneous field.

These results indicate that the the growth of quantum fluctuations of the inflaton sector fields to form non-topological solitons can be rapid in realistic D-term inflation models, occuring in not much more than the time for a single coherent oscillation of the inflaton field. This is consistent with a tachyonic preheating picture of perturbation 
growth [7] rather than an inflaton condensate fragmentation interpretation [6].

We next consider the case of fixed $\lambda / g=0.14$ and investigate the effect of the variation of the mode $k$ on the growth of the perturbations. Figure 5 shows the transition from a) the case of almost no-growth at $k=0.001 s_{c}$ to b) fully non-linear growth $(\delta s / s(t) \gtrsim 1.0)$ at $k \approx 0.005 s_{c}$. In Figure 6 from a) to b) a dramatic damping in the growth of the perturbation is seen as $k$ increases above $0.01 s_{c}$. Since the effect of large gradient terms in the equations of motion is to damp the growth of the perturbation, this would indicate that we have passed the maximum value, $k_{\max }$, for which growth of inflaton perturbations is possible. The dominant mode for a given $\lambda / g$ will correspond to that which grows to become fully non-linear first, which will correspond to $k \approx k_{\max }$.

In Figures 7 and 8 we consider $g=0.5$ whilst keeping $\lambda / g=0.14$. Again we vary $k$ to show the transition from no growth to fully non-linear growth. In Figure $7 \mathrm{~b}$ ) we find that for $k=0.001 s_{c}$ the mode can grow to become non-linear but in a time much longer than the period of coherent oscillations. Growth of this mode would be well described by the inflaton condensate fragmentation approach [6]. However, it is the mode which grows most rapidly that will reach non-linearity first and so determine the length scale of the condensate lumps. Typically this dominant mode reaches non-linearity in a few oscillations.

Comparing Figure 8a) with Figure 6a), we see that exactly the same maximum growth of the perturbation is obtained at $k$ close to $k_{\max }$ for both $g=0.5$ and $g=1.0$ when $\lambda / g$ is fixed. This shows that the maximum growth of the perturbations is dependent only upon the ratio $\lambda / g$. We also see that $k_{\max } \approx 0.007 s_{c}$ for $g=0.5$, compared with $k_{\max } \approx 0.015 s_{c}$ for $g=1$, indicating that $k_{\max } \propto g$ for fixed $\lambda / g$.

An important quantity is the lower limit on $\lambda / g$ for which the perturbations can

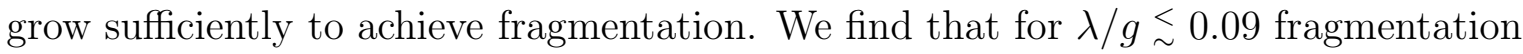
does not occur for any value of $k$. Thus for $\lambda / g \gtrsim 0.09$ we expect fragmentation into non-topological solitons to occur in D-term inflation. The value of $k_{\max }$ corresponding to the limiting case $\lambda / g \approx 0.09$ is $k_{\max } \approx 0.008 s_{c}$.

Although the above results strictly apply to the case of D-term inflation, we note 


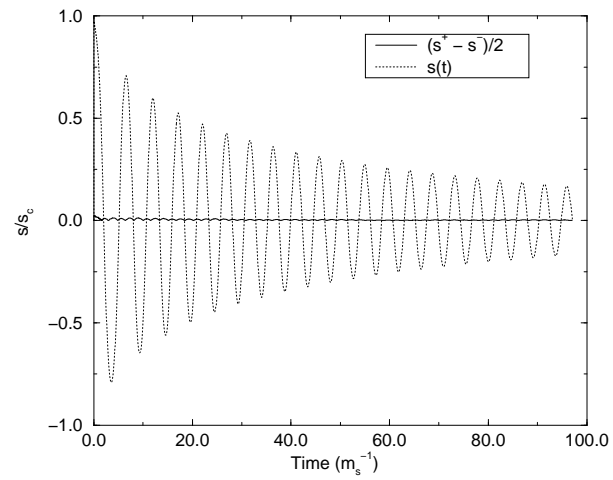

(a) $\frac{\lambda}{g}=0.10$

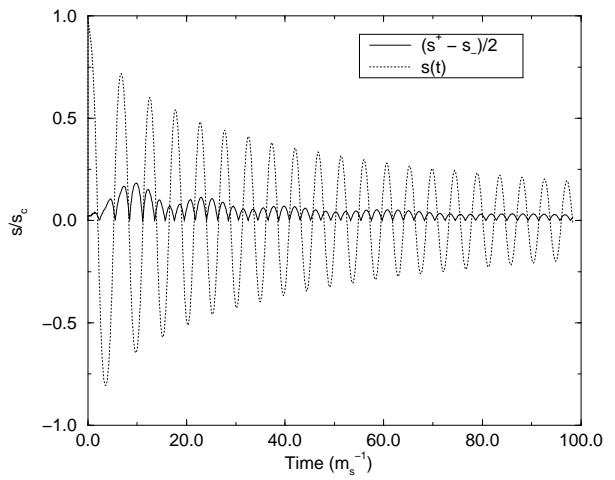

(b) $\frac{\lambda}{g}=0.12$

Figure 3: $k=0.01 s_{c}$. As we increase $\frac{\lambda}{g}$ from 0.10 to 0.12 we start to see growth in the perurbation amplitude though it is still well within the linear regime.

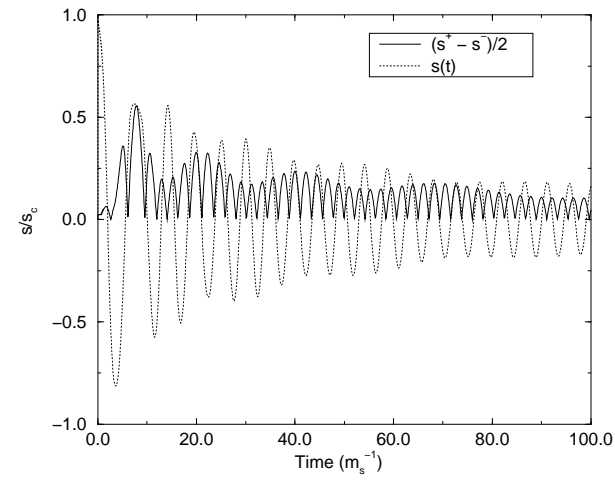

(a) $\frac{\lambda}{g}=0.14$

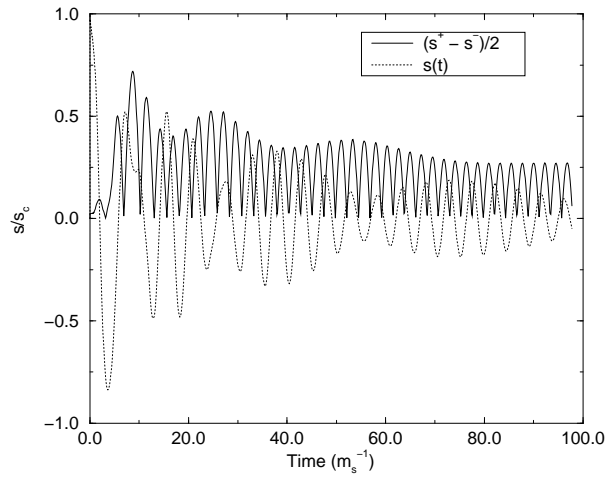

(b) $\frac{\lambda}{g}=0.2$

Figure 4: $k=0.01 s_{c}$. (a) shows the critical value of $\frac{\lambda}{g}$ for which the perturbation just becomes fully non-linear. Any further increase ((b)) pushes the perturbation deeper into the non-linear regime. 
that in the limit where the classical potential becomes identical to F-term inflation, $\lambda=\sqrt{2} g$, condensate fragmentation is certain to occur in D-term inflation. This strongly suggests that fragmentation will be an inevitable feature of F-term inflation models.

It is interesting to compare these results with the analytical results obtained in the inflaton condensate fragmentation approach [6]. Although the approximations made in [6] are not valid for perturbations which grow rapidly on the time scale of the coherent oscillations, we nevertheless find that the dependence of $k_{\max }$ and the fragmentation condition on $\lambda$ and $g$ are well described by the analytical expressions in [6]. $k_{\max }$ is given by

$$
\frac{\sqrt{3} \lambda^{2}}{2 g} s_{c},
$$

where we have used $R=s_{c}$ and $a_{o}=a$ in Equation 29 of [6], consistent with $k_{\text {max }}$ at the onset of coherent oscillations. Thus we find $k_{\max } \propto g$ for fixed $\lambda / g$. For $\lambda / g=0.14$ we find from Eq. (36) that $k_{\max }=0.008 s_{c}\left(0.016 s_{c}\right)$ for $g=0.5(1.0)$, in reasonable agreement with our numerical results. The condition for fragmentation to occur from the inflaton condensate fragmentation analysis is [6]

$$
\frac{\lambda}{g} \gtrsim \frac{4 \sqrt{2} \xi^{1 / 2} \beta}{M} \approx 0.2\left(\frac{\beta}{10}\right),
$$

where $\beta=\log \left(s_{c} / \delta s\left(t_{o}\right)\right)$. This depends only on the ratio $\lambda / g$, in agreement with the numerical analysis. (The actual value of the lower bound from Eq. (37) can only be taken as suggestive, as the various approximations made introduce 'factor of 2' uncertainties.) We also note that the rate of growth of perturbation from the inflaton condensate fragmentation analysis (given by $\alpha$ (Equation 27) in [6]) is proportional to $k$ for values up to $k_{\max }$, beyond which there is no perturbation growth, in qualitative agreement with the numerical results.

The broad agreement of the numerical $k_{\max }$ with the inflaton condensate fragmentation expression suggests that the exponential terms governing the growth of perturbations have the same parameter dependence in the realistic case as in the inflation condensate fragmentation analysis, although there is no reason to expect the 
perturbation growth at $k \approx k_{\max }$ to be quantitatively well-described by this analysis.

Finally, we would like to comment on the possible consequences of including the $\Phi_{+}$field in the analysis. During inflation $m_{\Phi_{+}} \geq \sqrt{2} g \xi^{1 / 2} \gg H$. Therefore we expect that the homogeneous field will satisfy $\Phi_{+}=0$ at the end of inflation. In this case the homogeneous $\Phi_{+}$field will not evolve since $\Phi_{+}=0$ is an extremum of the potential for all values of $S$ and $\Phi_{-}$. A quantum fluctuation of $\Phi_{+}$could grow if the mass squared of $\Phi_{+}$at $\Phi_{+}=0$ were to become negative during the post-inflation evolution of the $S$ and $\Phi_{-}$fields. This could briefly occur as $|S|$ approaches zero if the value of the oscillating $\Phi_{-}$field was also sufficiently larger than its minimum as a function of $|S|$ at this time. However, the initial quantum de Sitter fluctuation of $\Phi_{+}$will be suppressed by its large mass compared with $H$ during inflation. In addition, the brief growth as $|S|$ passes through zero will result in only a small growth of the quantum fluctuation of $\Phi_{+}$. This is particularly true since the dominant $S$ modes reach fragmentation within only a few oscillations of the homogeneous $S$ field. Therefore we expect that the $\Phi_{+}$ field will have a negligible effect on the evolution of the energy density during the post-inflation era and that we may consider $\Phi_{+}=0$ throughout. 

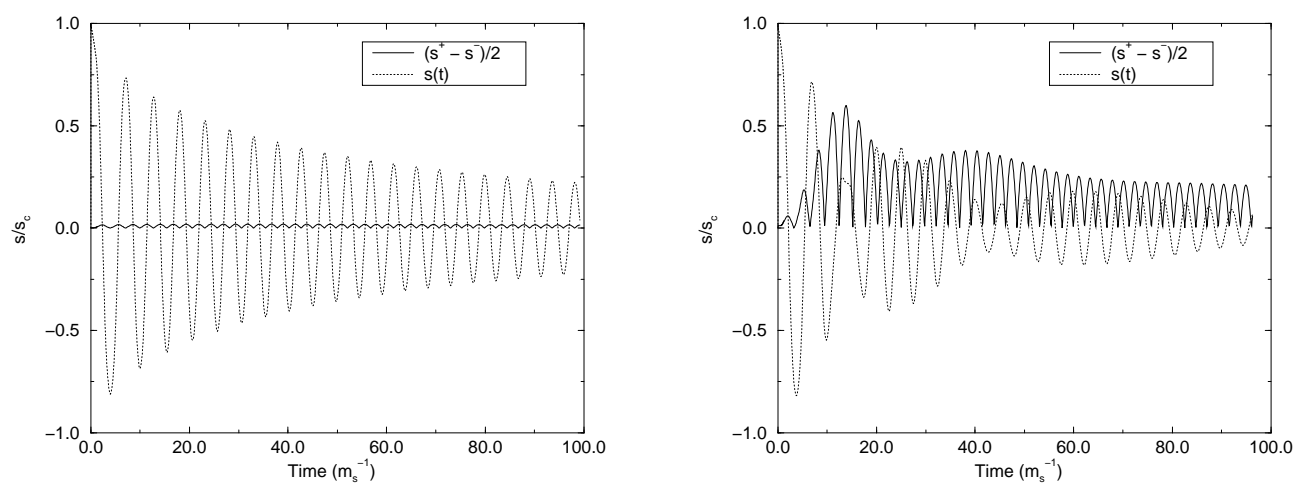
(a) $k=0.001 s_{c}$
(b) $k=0.005 s_{c}$

Figure 5: $\frac{\lambda}{g}=0.14, g=1$. As expected, for a very small value of $k((\mathrm{a}))$ there is no growth in the perturbation as the gradient term in the equations of motion are negligable. As $k$ is increased ((b)) we get the expected evolution into the non-linear regime.

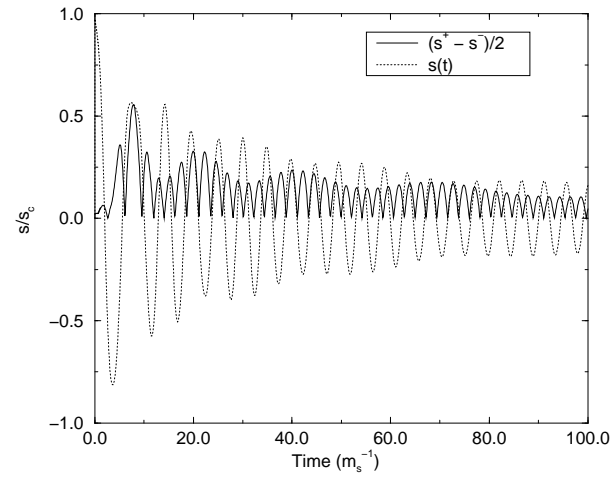

(a) $k=0.010 s_{c}$

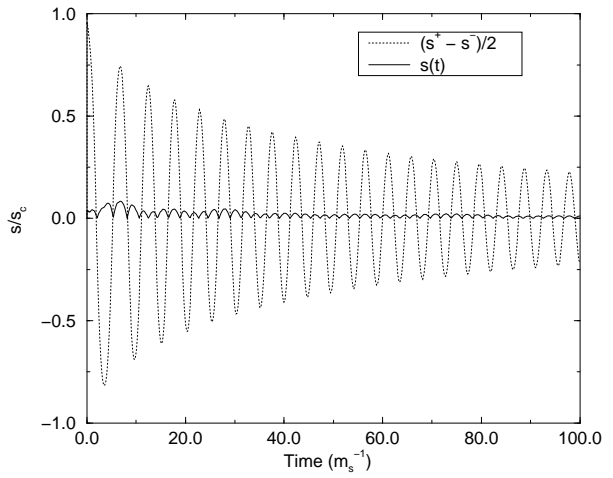

(b) $k=0.015 s_{c}$

Figure 6: $\frac{\lambda}{g}=0.14, g=1$. Further increase ((a)) gives us the maximum value of $k$ for which full non-linearity occurs earliest at this value of $\frac{\lambda}{g}$. This corresponds to the dominant mode leading to fragmentation. Finally, (b) illustrates the case $k \approx k_{\max }$ with suppressed perturbation growth. 


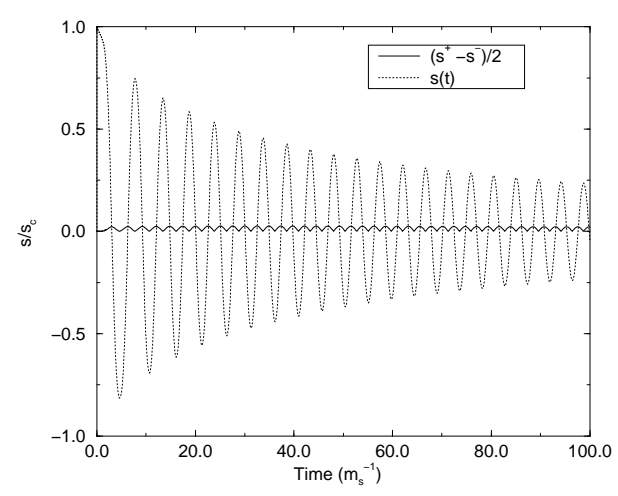

(a) $k=0.0005 s_{c}$

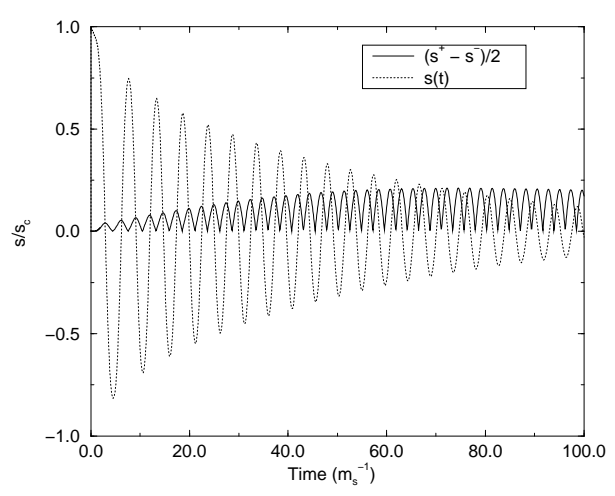

(b) $k=0.001 s_{c}$

Figure 7: $\frac{\lambda}{g}=0.14, g=0.5$. As before we see the same increase in the rate of perturbation growth as we increase the initial mode size $k$. (b) shows a mode with slow growth towards full non-linearity.

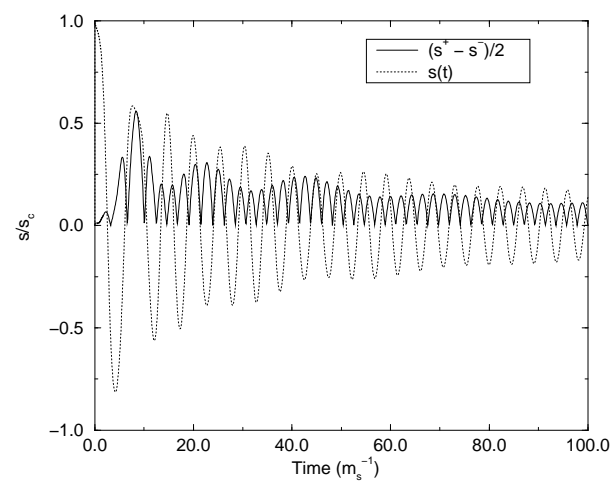

(a) $k=0.005 s_{c}$

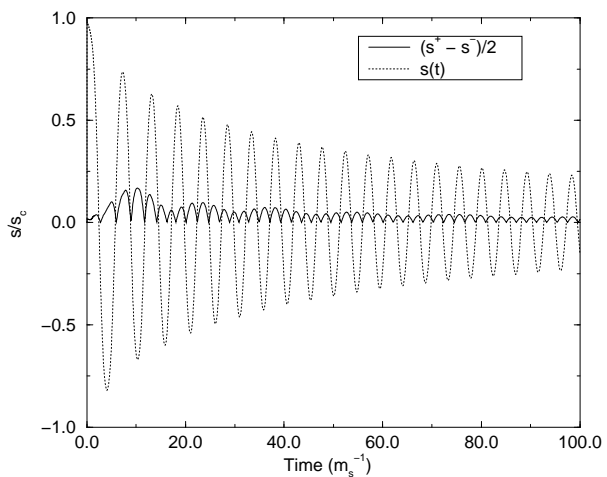

(b) $k=0.007 s_{c}$

Figure 8: $\frac{\lambda}{g}=0.14, g=0.5$. (a) shows the limiting case with $k \approx k_{\max }$ where the perturbation just reaches full non-linearity. Further slight increase in the mode size to $k \geq k_{\max }((\mathrm{b}))$ suppresses growth in the perturbation as before. 


\section{Consequences of Inflatonic Non-Topological Soli- ton Formation}

In this section we discuss some possible cosmological consequences of non-topological soliton (NTS) formation. After fragmentation the inflaton sector fields are expected to form NTS which we have labelled inflaton condensate lumps [6], with essentially all of the energy density of the Universe concentrated in the lumps [6, 9]. Physically these

are interpreted as a Bose condensate of scalars held together by an attractive selfinteraction which balances against the gradient terms (uncertainty principle pressure) in the scalar field equations. The most obvious physical consequences of inflaton condensate lump formation will be related to the inhomogeneous distribution of the energy density of the Universe in the post-inflation NTS era and the inhomogeneous nature of reheating via NTS decay.

The decay rate of the condensate lumps is related to (i) its stability as a solution of the classical equations of motion and (ii) the decay and annihilation rate of the scalars inside the NTS. For the case of a real scalar field it is likely that the condensate lumps are not stable, but instead slowly radiate scalar field waves until (depending on the potential) the amplitude of the scalar field inside the lumps becomes too small for the attractive interaction term to hold the lumps together. This has been studied extensively in [22], where the condensate lumps have been given the name 'oscillons'. The lifetime of the oscillons in a potential of the form $V(\phi)=m^{2} \phi^{2} / 2-\lambda \phi^{4} / 4$ (which approximates D-term inflation [6]) has been estimated to be around $10^{3-4} m^{-1}[22$, 8]. In this case we would expect the scalars forming the lump to eventually disperse. However, during the period when the condensate lumps exist, the fact that the number density of scalars inside the lumps is constant (unlike the case of a conventional homogeneous inflaton condensate [4]) means that perturbative inflaton annihilation and/or parametric resonance will be effectively enhanced and could dominate over perturbative inflaton decays as the main mode of reheating [6]. This all depends on whether the classical decay of the condensate lumps is slow enough relative to annihilation/parametric resonance for the condensate lumps to have non-trivial consequences 
for reheating.

However, an important point is that in SUSY hybrid inflation models the inflaton is in fact a complex field. In D-term inflation there is a global $U(1)_{S}$ symmetry such that $S \rightarrow e^{i \alpha} S$ and $\Phi_{+} \rightarrow e^{-i \alpha} \Phi_{+}$in the superpotential. As a result, there will be a Q-ball solution of the inflaton sector equations of motion [23] carrying a non-zero $U(1)_{S}$ charge. In this case it is likely that the initially neutral condensate lump will fragment into a \pm Q-ball pair 24]. This occurs because although initially the inflaton field and condensate lump is real, small fluctuations of the phase of the inflaton field inside the lump can grow through mode-mode couplings, resulting in a seperation of charge in the quasi-stable condensate lump into a Q-ball, anti-Q-ball pair. This has been demonstrated in lattice simulations of a running mass chaotic inflation model 24]. Although the potential of this single-field inflation model is different from that of D-term inflation, we would expect the tendency of the system to reach its most stable configuration ( \pm Q-ball pairs) in the presence of spatial perturbations to be repeated.

Since the Q-balls are classically stable, reheating will be determined by the decay/annihilations of the globally charged inflaton sector fields forming the Q-balls i.e. reheating will occur via Q-ball decay, with the energy density of the Universe concentrated inside the classically stable Q-balls until that time. Therefore reheating and thermalisation will be highly inhomogeneous in this case, unlike the case of a conventional homogeneous inflaton condensate.

The formation of condensate lumps and fragmentation to Q-balls also results in a highly inhomogeneous energy density prior to reheating. One consequence of this is that the dynamics of scalar fields in SUSY and SUGRA cosmology will be altered. In SUGRA models, Planck-scale suppressed corrections result in a correction to the mass squared term of scalar fields the form $|F|^{2} / M_{P l}^{2} \approx \rho / M_{P l}^{2}$, where $F$ represents the F-term of any field in the cosmological background and $\rho$ is its the energy density. In the conventional case of a homogeneous coherently oscillating inflaton field following inflation, $\rho$ is also homogeneous and so the correction to the mass squared term is of the form $\bar{\rho} / M_{P l}^{2} \sim c H^{2}$, where $\bar{\rho}$ the average energy density and $|c|$ is of the order of 1 [14. However, when the energy density is packed into Q-balls, the correction to 
the mass squared term of the scalars outside the Q-balls will be effectively zero, whilst inside it will be much larger than $H^{2}$. As a result, the dynamics of flat direction scalars in the MSSM [25] (and other so-called moduli fields) will be completely different from the conventional case. In the bulk outside the Q-balls (corresponding to most of the volume of the Universe) the scalar fields will not evolve until $H^{2} \approx m_{\text {susy }}^{2}$ (which occurs prior to reheating if the reheating temperature satisfies the thermal gravitino constraint $T_{R} \curvearrowright 10^{8} \mathrm{GeV}[26]$, where $m_{\text {susy }}^{2} \approx(100 \mathrm{GeV})^{2}$ is the conventional gravity-mediated SUSY-breaking mass squared term. Inside or close to the Q-balls, if we consider the mass squared correction due to $\rho$ to be positive, the flat direction scalar will be driven towards zero. The full dynamics of the MSSM flat directions will require a complete solution of the scalar field equations in the presence of Q-balls, but we expect the dynamics in the bulk to be essentially that of $c=0$ in the conventional discussion of MSSM flat directions and moduli. This will have significant consequences for the cosmology of the MSSM, in particular for Affleck-Dine baryogenesis [27, 25]. We expect other consequences of the NTS dominated post-inflation era to become apparent in the future.

\section{Conclusions}

We have analysed the growth of spatial perturbations of the inflaton sector fields of D-term hybrid inflation including 1-loop radiative corrections, using a semi-analytic model with simplified initial conditions. We have argued that this model will give a good estimate of the growth of spatial perturbation modes in the fully realistic case.

We find that the growth of the perturbations to fully non-linearity (indicating the onset of inflaton condensate lump formation) occurs once $\lambda / g \gtrsim 0.09$, and that the maximal growth factor of the perturbations is a function only of $\lambda / g$. Therefore non-topological soliton formation is a natural possibility in D-term inflation models. The corresponding perturbation mode, which sets the length scale for the condensate lumps in the case where $\lambda / g \approx 0.09$, is given by $k \approx 0.008 s_{c}$ for $g=1$, with $k \propto g$ for fixed $\lambda / g$. The growth of spatial perturbations to full non-linearity is rapid, typically 
after about one coherent oscillation of the inflaton field, which is consistent with a tachyonic preheating picture [7] of the growth of spatial perturbations. However, the value of $k_{\max }$, the largest wavenumber for which perturbation growth can occur, and the dependence of the fragmentation condition only on the ratio $\lambda / g$ are both consistent with the analytical predictions of the inflaton condensate fragmentation analysis [6].

Although our analysis strictly applies to the case of D-term inflation, it strongly suggests that inflaton condensate fragmentation will be an unavoidable feature of Fterm hybrid inflation, which has the same classical scalar potential as D-term inflation in the limit $\lambda=\sqrt{2} g$. However, a full analysis including the 1-loop potential of F-term inflation remains to be done.

The formation of non-topological solitons (inflaton condensate lumps) is undoubtedly a feature of the cosmology of SUSY hybrid inflation models for a natural range of the inflaton sector couplings, resulting in a highly inhomogeneous post-inflation era with all the energy of the Universe packed inside the non-topological solitons. We have discussed some possible consequences of inflaton condensate lump formation for SUSY cosmology, including the formation of inflatonic Q-balls from decay of the condensate lumps, inhomogeneous reheating and the modification of flat direction scalar field dynamics. We hope to develop a more detailed understanding of this post-inflation era in the future.

\section{Acknowledgements}

The research of M.B. was supported by the EPSRC.

\section{References}

[1] E.W.Kolb and M.S.Turner, The Early Universe, (Addison-Wesley, Reading, MA, 1990). 
[2] J.H.Traschen and R.H.Brandenberger, Phys. Rev. D42 (1990) 2491; A.D.Dolgov and D.P.Kirilova, Sov. J. Nucl. Phys. 51 (1990) 172.

[3] S.Y.Khlebnikov and I.I.Tkachev, Phys. Rev. D56 (1997) 653; L.Kofman, A.D.Linde and A.A.Starobinsky, Phys. Rev. D56 (1997) 3258.

[4] I.Zlatev, G.Huey and P.J.Steinhardt, Phys. Rev. D57 (1998) 2152.

[5] A.Linde, Phys. Rev. D49 (1994) 748.

[6] J.McDonald, Phys. Rev. D66 (2002) 043525.

[7] G.Felder, J.Garcia-Bellido, P.B.Greene, L.Kofman, A.Linde and I.Tkachev, Phys. Rev. Lett. 87 (2001) 011601; G.Felder and L.Kofman, Phys. Rev. D63 (2001) 103503; G.Felder, L.Kofman and A.Linde, Phys. Rev. D64 (2001) 123517.

[8] S.Kasuya, M.Kawasaki and F.Takahashi, hep-ph/0209358.

[9] E.J.Copeland, S.Pascoli and A.Rajantie, Phys. Rev. D65 (2002) 103517.

[10] K.Enqvist, S.Kasuya and A.Mazumdar, Phys. Rev. Lett. 89 (2002) 091301; Phys. Rev. D66 (2002) 043505.

[11] G.Dvali, Q.Shafi and R.Schaefer, Phys. Rev. Lett. 73 (1994) 1886.

[12] E.Halyo, Phys. Lett. B387 (1996) 43; P.Binetruy and G.Dvali, Phys. Lett. B388 (1996) 241.

[13] A.D.Linde, Phys. Lett. B129 (1983) 177.

[14] M.Dine, W.Fischler and D.Nemeschansky, Phys. Lett. B136 (1984) 169; G.D.Coughlan, R.Holman, P.Ramond and G.G.Ross, Phys. Lett. B140 (1984) 44; O.Bertolami and G.G.Ross, Phys. Lett. B183 (1987) 163; E.J.Copeland, A.R.Liddle, D.H.Lyth, E.D.Stewart and D.Wands, Phys. Rev. D49 (1994) 6410; M.Dine, L.Randall and S.Thomas, Phys. Rev. Lett. 75 (1995) 398; G.Dvali, Phys. Lett. B355 (1995) 78. 
[15] J.Lesgourgues, D.Polarski and A.A.Starobinsky, Nucl. Phys. B497 (1997) 479; C.Kiefer, D.Polarski and A.A.Starobinsky, Int.J.Mod.Phys D7 (1998) 455.

[16] D.Lyth and A.Riotto, Phys. Rep. 314 (1999) 1.

[17] R.Jeannerot and J.Lesgourgues, Phys. Rev. D62 (2000) 023514.

[18] E.J.Weinberg and A.Wu, Phys. Rev. D36 (1987) 2474.

[19] A.Guth and S-Y.Pi, Phys. Rev. D32 (1985) 1899.

[20] K.Enqvist and J.McDonald, Phys. Lett. B425 (1998) 309; Nucl. Phys. B538 (1999) 321.

[21] S.Kasuya and M.Kawasaki, Phys. Rev. D61 (2000) 041301; Phys. Rev. D62 (2000) 023512.

[22] M.Gleiser and A.Sornborger, Phys. Rev. E62 (2000) 1368; E.J.Copeland, M.Gleiser and H.-R.Muller, Phys. Rev. D52 (1995) 1920.

[23] M.Broadhead and J.McDonald, In preparation.

[24] K.Enqvist, S.Kasuya and A.Mazumdar, Phys. Rev. D66 (2002) 043505.

[25] K.Enqvist and A.Mazumdar, hep-ph/0209244

[26] J.Ellis, J.E.Kim and D.V.Nanopoulos, Phys. Lett. B145 (1984) 181.

[27] I.A.Affleck and M.Dine, Nucl. Phys. B249 (1985) 361. 